\title{
BMJ Open Effect of salt reduction interventions in lowering blood pressure in Chinese populations: a systematic review and meta-analysis of randomised controlled trials
}

Aoming Jin (D) , ${ }^{1}$ Wuxiang Xie, ${ }^{1}$ Yangfeng $\mathrm{Wu}^{1,2}$

To cite: Jin A, Xie W, Wu Y. Effect of salt reduction interventions in lowering blood pressure in Chinese populations: a systematic review and meta-analysis of randomised controlled trials. BMJ Open 2020;10:e032941. doi:10.1136/ bmjopen-2019-032941

- Prepublication history and additional material for this paper are available online. To view these files, please visit the journal online (http://dx.doi. org/10.1136/bmjopen-2019032941).

Received 16 July 2019 Revised 22 January 2020 Accepted 23 January 2020

Check for updates

(c) Author(s) (or their employer(s)) 2020. Re-use permitted under CC BY-NC. No commercial re-use. See rights and permissions. Published by BMJ.

${ }^{1}$ Peking University Clinical Research Institute, Peking University First Hospital, Beijing, China

${ }^{2}$ Epidemiology, Peking University School of Public Health, Beijing, China

Correspondence to Dr Yangfeng Wu; wuyf@bjmu.edu.cn

\section{ABSTRACT}

Rationale and objective Salt reduction remains a global challenge and different salt reduction strategies have been studied in China. This study is to systematically evaluate evidence from randomised controlled trials (RCT) in China and inform the effective salt reduction strategies.

Design Systematic review and meta-analysis. Data sources MEDLINE, Embase, Cochrane Central Register of Controlled Trials, Wanfang Data and the China National Knowledge Infrastructure databases through October 2019

Eligibility criteria RCTs conducted in China with at least 4 weeks' duration of study and blood pressure (BP) reported.

Data extraction and synthesis Data were screened, extracted and appraised by two independent reviewers. The quality of study was assessed using a modified Cochrane Collaboration's risk of bias tool. The primary outcome was the difference in BP change from baseline to the end of study between interventions and control. The effects were pooled using a random effects model and associated factors were explored by a metaregression.

Results We identified 24 studies involving 10448 participants, including 8 studies on health education (4583 participants), 2 studies on salt restriction diet (162 participants), 1 study on salt restriction spoon (50 participants) and 13 studies on salt substitute (5653 participants). Six studies on salt substitute and three studies on health education were identified with high quality. Pooled results from the six studies showed that salt substitutes significantly reduced systolic BP $(-5.7 \mathrm{~mm}$ $\mathrm{Hg} ; 95 \% \mathrm{Cl}-8.5$ to -2.8$)$ and diastolic BP $(-2.0 \mathrm{~mm} \mathrm{Hg}$; $95 \% \mathrm{Cl}-3.5$ to -0.4$)$. The School-EduSalt study showed that the school-based health education significantly reduced systolic BP among parents $(-2.3 \mathrm{~mm} \mathrm{Hg} ; 95 \% \mathrm{Cl}$ -4.5 to -0.04$)$.

Conclusions Among four salt reduction strategies studied in China with RCT design, only salt substitute was proved effective in lowering BP by the pooled effect from multiple studies with high quality. More well-designed studies are warranted for other strategies.
Strengths and limitations of this study

- This systematic review evaluates salt reduction strategies on the effect of lowering blood pressure in Chinese population, which has a very high dietary salt intake and requires different strategies for population salt reduction.

- We included randomised controlled trials only and used a modified Cochrane Collaboration's risk of bias tool to evaluate the quality of each single study.

- Meta-regression was used to understand which strategies were more effective with adjustment for other associated factors.

- Because studies with high quality were limited mainly to salt substitutes, there was no capacity to draw conclusion on the difference between salt reduction strategies regarding their effect size.

\section{INTRODUCTION}

Hypertension is the most important risk factor of cardiovascular disease (CVD) morbidity and mortality, and is responsible for 211 million disability-adjusted life years worldwide each year. ${ }^{1}$ According to the Global Burden of Disease Study 2017, it was estimated that 17.8 million deaths, nearly one-third of the total, attributed to CVD, mainly ischaemic heart disease (IHD) and cerebrovascular disease. ${ }^{2}$ Moreover, approximately $54 \%$ of stroke and $47 \%$ of IHD were associated with high blood pressure (BP). ${ }^{3}$ Elevated BP has been recognised as a major threat to worldwide public health. ${ }^{4}$

Several national surveys during the last half of the century demonstrated that the prevalence of hypertension has been continually increasing in China, reaching at 23.2\% in $2015 .^{5}$ It would transform to 244.5 million adults suffering hypertension in this most populous country. Meanwhile, only $15.3 \%$ of 
them had their BP under control. ${ }^{5}$ Effective strategies for control of hypertension are critically needed.

The effect of salt reduction in lowering BP has been proved in randomised controlled trials (RCT). ${ }^{6}$ Given the strong scientific evidence, salt reduction has been recommended by the WHO as a highly cost-effective 'best buy' for control and prevention of hypertension and CVD globally. ${ }^{7}$ However, how to reduce salt intake effectively is still a global-wide challenge. Many populations in the world are still having a daily salt intake level that is much higher than the WHO recommended $5 \mathrm{~g}$ /day, including China. ${ }^{8}$ However, majority of sodium intake of Chinese was from home-based cooking. ${ }^{9}$ Compulsory or voluntary salt reduction programmes with food industry that were proved effective in developed countries are not the priority. There have been different intervention strategies such as salt substitute, health education, salt restriction diet and salt restriction spoon being employed in China, and many of them were evaluated with randomised trials. The present study aims to systematically review different salt reduction intervention strategies that were tested through RCTs conducted in China, in order to provide scientific evidence on the effectiveness of these strategies in lowering BP, and to inform the current public health policies.

\section{MATERIALS AND METHODS}

\section{Eligibility criteria for included studies}

Studies were included in the review if they met the following inclusion criteria: (1) using RCT design; (2) the intervention tested aiming to reduce salt intake; (3) being conducted in Chinese population; (4) duration of intervention was at least 4 weeks; and (5) BP data were reported. To be included in the meta-analysis, the studies should also report the mean difference and SD in changes in BP from baseline to the end of follow-up between the intervention and control groups.

\section{Search strategy}

A systematic literature search was conducted in three major English databases (MEDLINE, Embase, Cochrane Central Register of Controlled Trials) and two major Chinese databases (Wanfang Data and China National Knowledge Infrastructure) by one study investigator (AJ). Each database was searched from inception to the end of October 2019. Our search combined free-test terms and keywords related to salt reduction interventions and BP control. We restricted the language of studies to English and Chinese. The full search strategy was presented in online supplementary appendix 1 .

\section{Study selection and data extraction}

Two reviewers (AJ and YW) independently identified studies for eligibility based on the inclusion and exclusion criteria. After all duplicates were removed, titles and abstracts of publications resulting from search strategy were initially screened. Potentially eligible publications were retrieved and the full-text version was reviewed in details. Disagreements were resolved by discussion. A data extraction sheet was developed to collect key information from each study, including characteristics of the study (authors, published year, sample size), the study participants (age, sex, baseline BP level and hypertension status), the intervention strategy (type, duration and fidelity) and outcome measures (pre/postintervention BP and sodium intake). Data were extracted independently by two study investigators (AJ and $\mathrm{YW})$. Discrepancies were resolved through discussion.

\section{Study quality and publication bias assessment}

The quality of the included studies was assessed by two study investigators (AJ and $\mathrm{YW}$ ) independently, adopting the Cochrane Collaboration's risk of bias tool. ${ }^{10}$ We modified it by adding two additional items, one on the intervention fidelity (ie, the evidence of salt reduction) and the other on the clarity of information in use of antihypertension medication, since we believe that information on these two items is also critical to our particular research interests. For the assessment of the risk of bias, we classified the studies into 'high', 'low' or 'unclear' risk categories according to the possible biases identified in the following nine methodological domains including random sequence generation, allocation concealment, blinding of participants and personnel, blinding of outcome assessment, incomplete outcome data, selective reporting, intervention fidelity, information for antihypertension medication use and other bias. The specific criteria for judging risk of bias of each domain were displayed in the online supplementary appendix 2. Disagreements were resolved by discussions. To be scored high quality, a study had to have at least seven out of nine methodology domains scored at 'low risk'. In addition, the following five methodology domains must all be scored at 'low risk': random sequence generation, allocation concealment, blinding of participants and personnel, intervention fidelity and information for antihypertension medication use.

\section{Statistical analysis}

The primary outcome was the change in BP from baseline to the end of intervention. The DerSimonian and Laird random effects models were used to estimate the pooled effect size and $95 \%$ CI because of wide methodological variability between the studies. Statistical heterogeneity between studies was quantified by means of $\mathrm{I}^{2}$ statistic. Trials with an $\mathrm{I}^{2}$ statistic $>50 \%$ indicated significant heterogeneity.

We first summarised the overall mean effect of salt reduction in studies with high quality only and further in all studies identified with meta-analysis. Then, we summarised the mean effect separately for studies with different salt reduction strategies, that is, health education, salt restriction diet, salt restriction spoon and salt substitute, with the same strategy as that for the overall effect. We did sensitivity analysis on the overall effect as 
well as the effect of different salt reduction strategies, by the leave-one-out method among studies with high quality only, to see if our results were affected by any particular trial. Furthermore, a meta-regression was conducted to confirm the association of the intervention effect in BP change with type of intervention strategy, with consideration of other trial characteristics including quality of study and hypertension status. Publication bias was assessed by evaluating funnel plots for asymmetry both visually and formally with Egger's test when there were at least 10 studies in the meta-analysis. ${ }^{11}$ All of the analyses were done by Stata software V.14.0 (StataCorp, College Station, TX, USA). A two-sided $p$ value $<0.05$ was considered statistically significant.

Patient and public involvement

No patient and public were involved in this study.

\section{RESULTS}

\section{Results of search}

In total, 3232 publications were initially identified from the database search. Six hundred and eighty-nine duplicates were removed, and the remaining 2543 articles were screened. After reviewing the titles and abstracts, 2470 articles were excluded. We retrieved the remaining 73 articles in full text, and 57 were further excluded due to not RCT design, no BP data reported, the intervention not just for salt reduction and the control group not blank. Finally, 16 articles fulfilled the inclusion and exclusion criteria. Among them, six articles reported multiple studies. Here, we defined a single study as a study with distinct study population or type of intervention strategy. Thus, a total of 24 studies with 10448 participants were included in our systematic review. Of those, three studies had no data on the change in BP from baseline to the end of intervention because only BP after intervention was reported. ${ }^{12} 13$ Therefore, our meta-analysis only included the remaining 21 studies (7720 participants). The detailed process of our literature search is summarised in the Preferred Reporting Items for Systematic Reviews and Meta-Analyses flow diagram in figure $1 .^{14}$

\section{Study characteristics}

The characteristics of the eligible trials are summarised in table 1 . Across all studies, 18 were individual RCT whereas six were cluster RCTs. These trials were published in years 2006-2019. The sample size of the trials ranged from 50 to 2566 . The study duration ranged from 2 months to 3 years. Among these studies, three included hospital patients with hypertension exclusively, eight included hypertensive participants from communities exclusively, nine enrolled a mix of hypertensive and normotensive participants, one enrolled participants at high risk of vascular disease, one enrolled normotensives exclusively, one in children and the other in the children's parents, respectively. All studies included both male and female participants except three unreported. The average age of

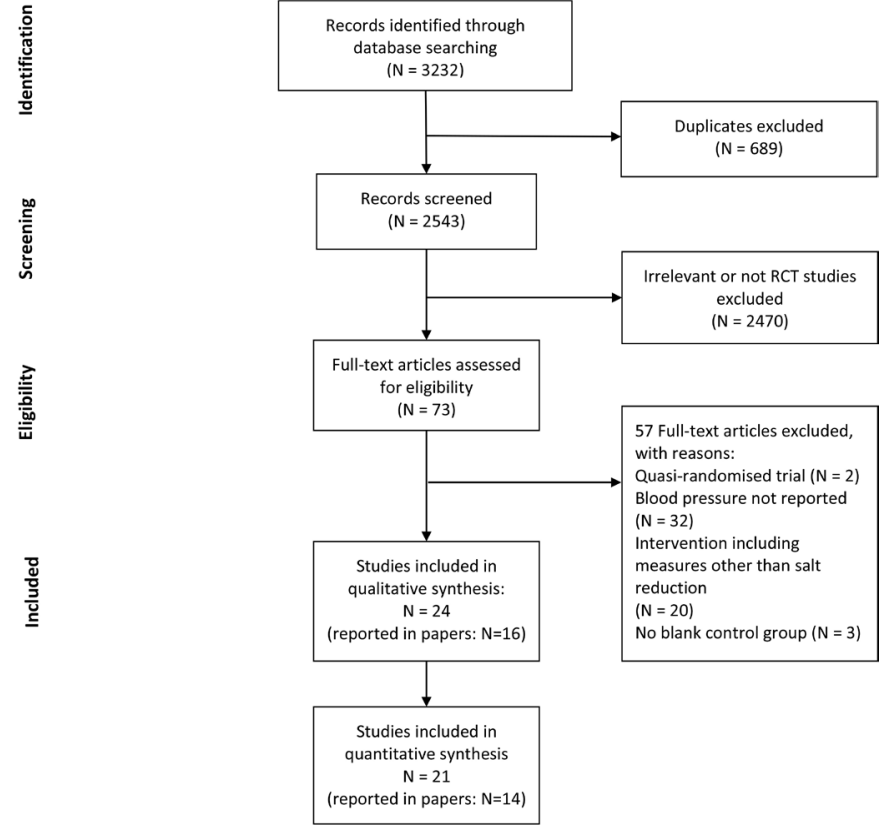

Figure 1 Flow diagram for study selection. RCT, randomised controlled trial.

enrolled participants varied from 10 to 69 years. In terms of intervention strategies used, out of the 24 studies, 8 used health education (from six articles), consisting of 4583 participants; 2 studies used salt restriction diet (from one article), consisting of 162 participants; 1 study used salt restriction spoon consisting of 50 participants; and 13 studies used salt substitute (from nine articles) with 5653 participants. Control groups received no active intervention. Three studies did not report the changes in BP from baseline to the end of intervention. ${ }^{12}{ }^{13}$ In addition, there were 17 studies that reported information on changes in sodium intake, either in 24 hours' urine, overnight urine, spot urine or weighting salt containers. Among eight studies employing 24 hours' urine collection, the median amount of sodium reduction in intervention groups compared with control groups was $-52.5 \mathrm{mmol} /$ day, ranging from -76 to $-14 \mathrm{mmol} /$ day.

\section{Quality assessment and publication bias}

The risk of bias assessment is summarised in figure 2 and online supplementary figure 1 . All trials had BP measured using standard devices, and hence should suffer no or little outcome assessment bias. And none of the trials reported data selectively. The most frequent biases were in the aspects of allocation concealment, participant blindness, fidelity of intervention and information on antihypertension medication use. Given the nature of the intervention, such as health education and salt restriction spoon, blinding of participants was not always adequately achieved in individual randomised trials. But many studies used cluster RCT design to increase the blindness and avoid contamination. The quality of studies on salt substitutes was much higher than that on the other salt reduction strategies, probably due to the easy blinding with such a nature of the intervention (figure 2). In total, 


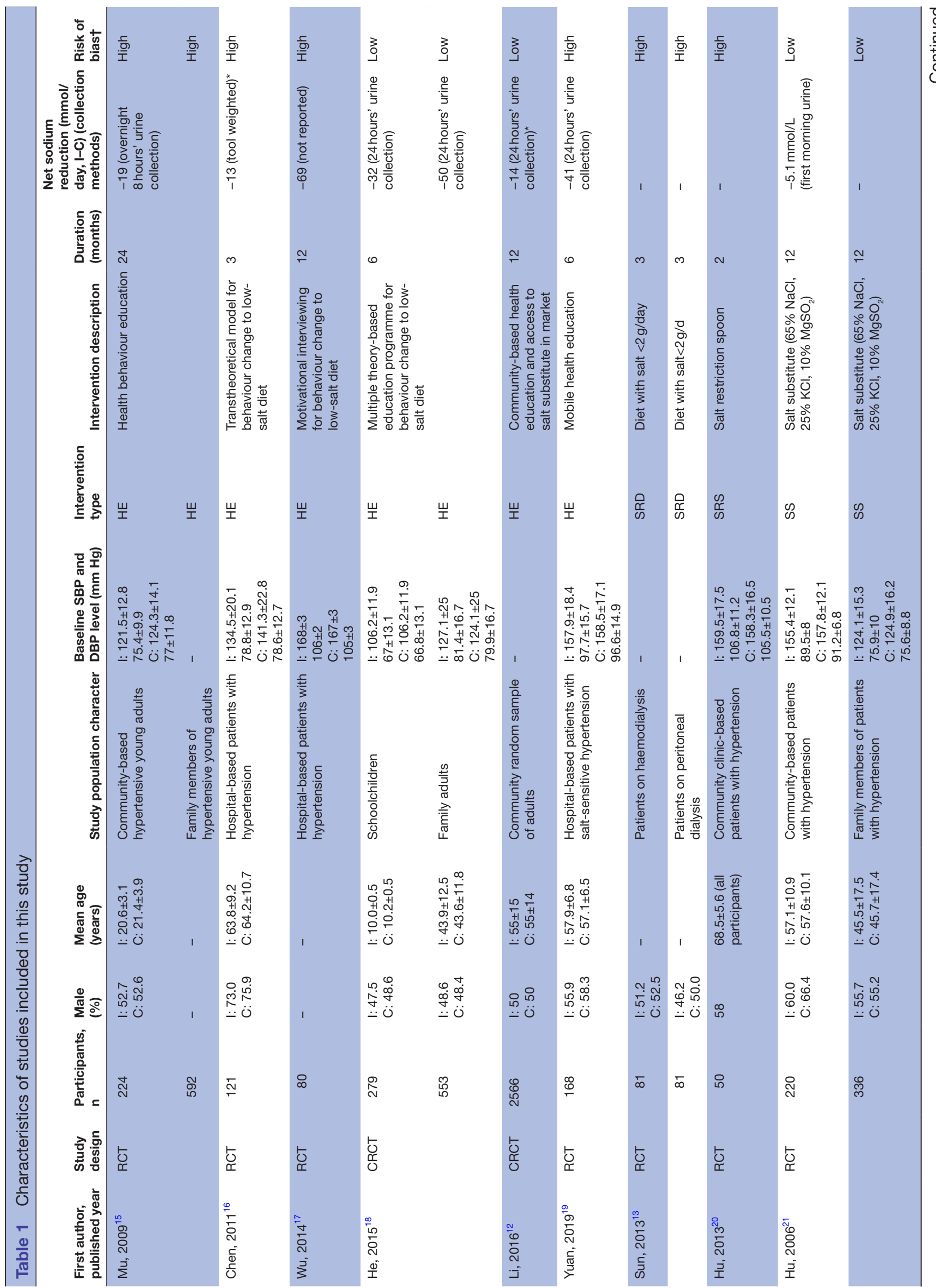




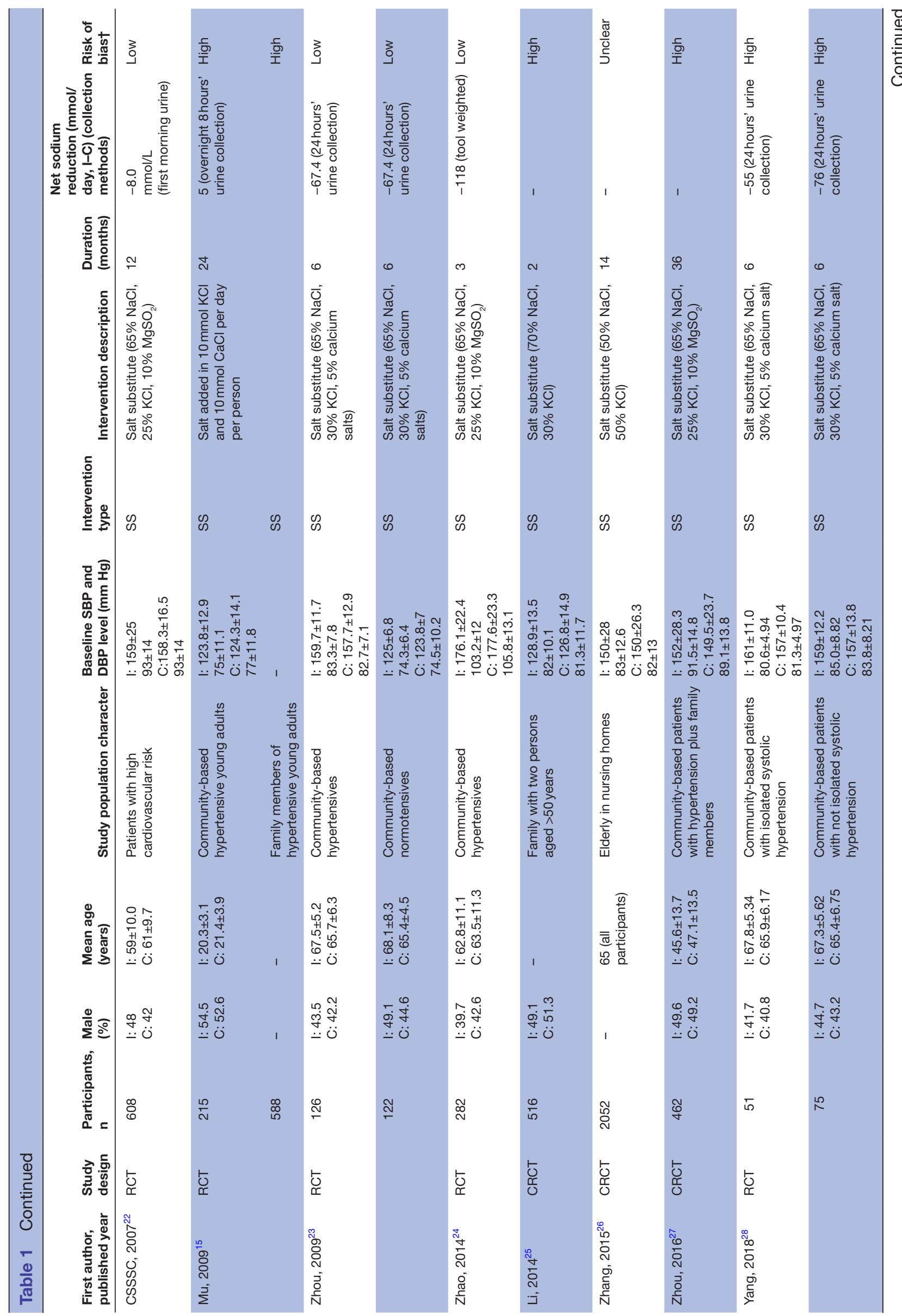




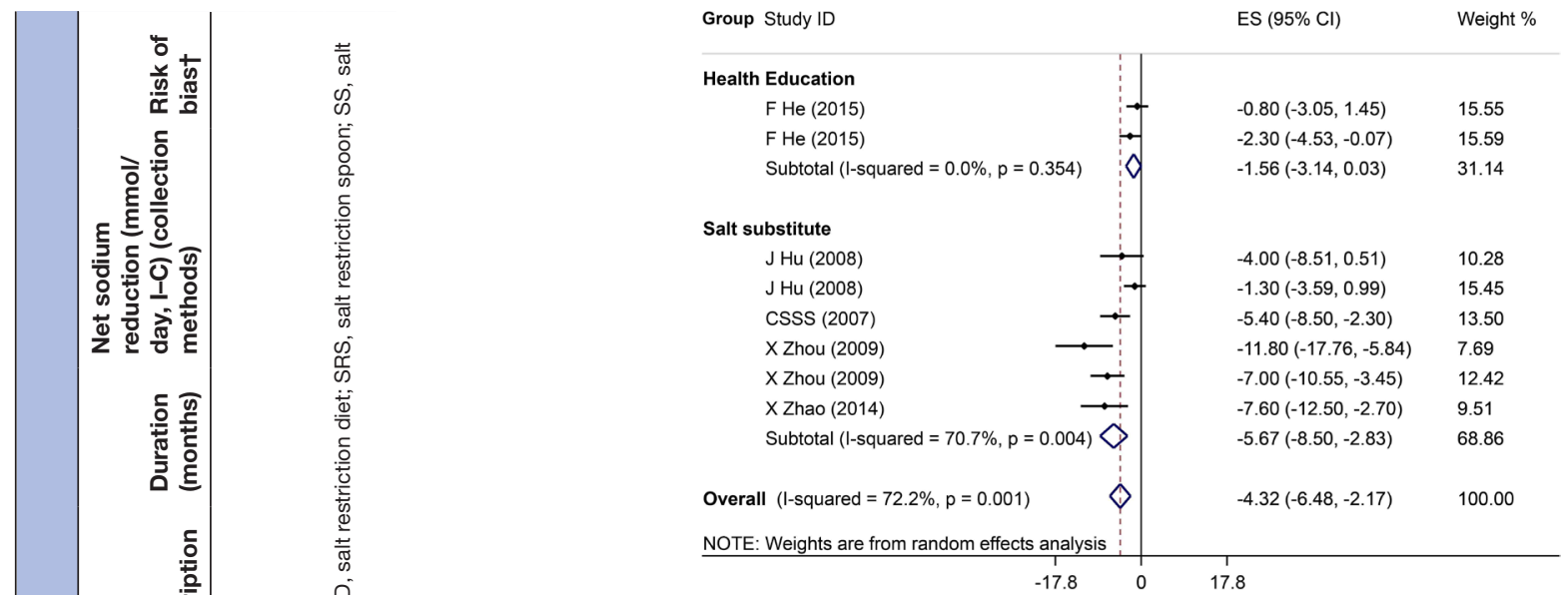

Figure 3 Forest plot of salt reduction interventions on effect in lowering systolic blood pressure among studies with high quality. ES, effect size.

there were nine studies with high quality, six on salt substitutes and three on health education (one of the three had no baseline outcome reported).

No evidence of publication bias was shown among all studies included by visually inspection of the funnel plot (online supplementary figure 2) or Egger's tests ( $\mathrm{p}=0.924$ and $\mathrm{p}=0.353$ for the effect on systolic and diastolic BP, respectively).

\section{Overall effect of salt reduction interventions}

The meta-analysis results of the reported effects of salt reduction interventions on BP are listed in figures 3 and 4. Analyses restricted to studies with high quality (six on salt substitute and two on health education) showed that the size of the overall mean intervention effect in lowering systolic BP was $-4.3 \mathrm{~mm} \mathrm{Hg} \mathrm{(95 \%} \mathrm{CI}-6.5$ to 2.2) and diastolic $\mathrm{BP}-1.6 \mathrm{~mm} \mathrm{Hg}(95 \% \mathrm{CI}-2.7$ to 0.4$)$, which became larger when all studies were included in the analysis $(-7.0 \mathrm{~mm} \mathrm{Hg}, 95 \% \mathrm{CI}-8.4$ to 5.7 for systolic BP; and $-3.6 \mathrm{~mm} \mathrm{Hg}, 95 \% \mathrm{CI}-4.5$ to 2.7 for diastolic BP).

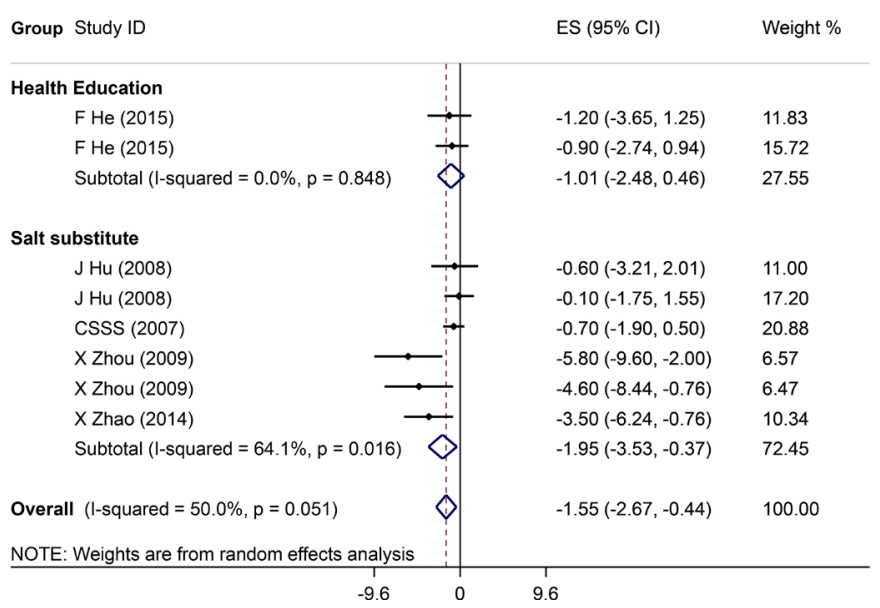

Figure 4 Forest plot of salt reduction interventions on effect in lowering diastolic blood pressure among studies with high quality. ES, effect size. 


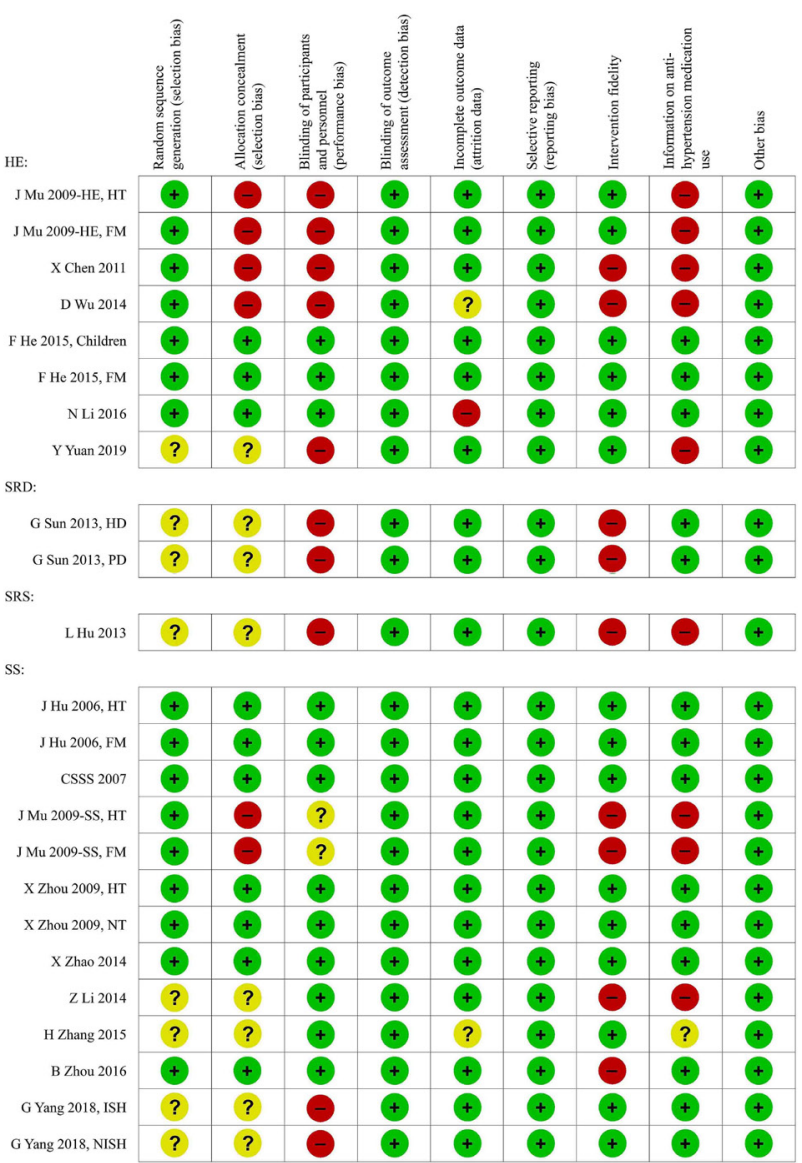

Figure 2 Risk of bias summary by study and methodological domain. FM, family members of hypertensives; HD, haemodialysis; $\mathrm{HE}$, health education; $\mathrm{HT}$, hypertensives; ISH, isolated systolic hypertensives; $\mathrm{NISH}$, not isolated systolic hypertensives; NT, normotensives; PD, peritoneal dialysis; SRD, salt restriction diet; SRS, salt restriction spoon; SS, salt substitute.

The sensitivity analysis that took the leave-one-out approach found that changes in the pooled effect estimates were minor.

\section{Effects of different salt reduction strategies \\ Health education for behaviour change}

There were eight studies using health education as salt reduction strategy in the review. Among them, the China Rural Health Initiative Salt Reduction Study (CRHI-SRS) used a cluster randomised controlled design with 60 villages on intervention and 60 villages on control. The intervention was community-wide health education plus social marketing of salt substitute. The results showed that the 24hours' urinary sodium excretion was significantly lower and potassium was significantly higher in the random sample of villagers in intervention villages than that in control villages at the end of 2 years' intervention. ${ }^{12}$ However, it was not appropriate to be included in the meta-analysis because (1) it did not report the baseline BP so that the change in BP was not available for our analyses; and (2) the study used two independent random samples of the villagers, which is very different from other trials that all used a cohort to measure the outcome. The remaining seven studies had data on BP change reported and were included in the meta-analysis. ${ }^{15-19}$ Six studies applied health education for behaviour changes to reduce daily salt intake. One disseminated health messages using mobile phones. ${ }^{19}$ The pooled effect sizes of the seven studies were quite large $(-8.1 \mathrm{~mm} \mathrm{Hg}$ in systolic BP and $-4.5 \mathrm{~mm} \mathrm{Hg}$ in diastolic BP). However, only two studies from the School-EduSalt trial scored high quality, which included schoolchildren and their parents but showed a much smaller effect than the pooled data. ${ }^{18}$ The study randomised 28 primary schools into a one-semester (16 weeks) school class-based health education programme on salt reduction or usual control. Two hundred and eighty grade 5 students and their family members (two for each student) were randomly selected for surveys before and after the intervention to evaluate the effect of the intervention. For all participants together, compared with the control group, the net reduction of 24 hours' urinary sodium excretion was about $25 \%$ in the intervention group. Specifically, the effect on BP for adults was $-2.3 \mathrm{~mm} \mathrm{Hg}$ in systolic $\mathrm{BP}(\mathrm{p}<0.05)$ and $-0.9 \mathrm{~mm} \mathrm{Hg}$ in diastolic BP $(p=0.31)$, and the corresponding effects were smaller and not statistically significant for children. ${ }^{18}$ Other studies all used an individual randomisation design but poorly reported concealment of allocation, did not blind the study participants and research staff and did not report information on antihypertension medication use. Two of them did not report information on intervention fidelity.

\section{Salt restriction diet}

Two studies from an article reported the effect of salt restriction diet, one in patients on haemodialysis and the other in patients on peritoneal dialysis. ${ }^{13}$ However, the study quality was not high. Details of the generation and concealment of the random allocation sequence as well as information on intervention fidelity were not reported and no measures were taken to blind participants and research staff. The baseline BP data were not reported and hence the effect sizes were not obtained. The postintervention data showed that systolic/diastolic BP was $158.1 / 88.3 \mathrm{~mm} \mathrm{Hg}$ in control group and 128.3/80.2 mm $\mathrm{Hg}$ in intervention group among patients on haemodialysis; the corresponding data were 142.1/98.4 and $123.3 / 91.7 \mathrm{~mm} \mathrm{Hg}$ among patients on peritoneal dialysis. $^{13}$

\section{Salt restriction spoon}

One RCT on salt restriction spoon was conducted. ${ }^{20}$ In the study, a salt restriction spoon with a volume to hold $2 \mathrm{~g}$ salt was designed to help housewives calculate the daily amount of salt they could use. The results showed that the use of salt restriction spoons could significantly reduce systolic and diastolic $\mathrm{BP}$ by 11.3 and $9.4 \mathrm{~mm} \mathrm{Hg}$, respectively. ${ }^{20}$ However, the quality of the study was poor because of insufficient information on the process of 
random sequence generation and allocation concealment, failure to blind participants and research staff and no data available regarding intervention fidelity and antihypertension medication use.

\section{Salt substitute}

Thirteen trials on salt substitution were included, the percentage of potassium chloride replacing sodium chloride ranged from $25 \%$ to $50 \% .^{1521-28}$ Six of those studies (1694 adults) were classified with high quality. ${ }^{21-24}$ Analyses restricted to those six with high quality showed a significant effect on $\mathrm{BP}(-5.7 \mathrm{~mm} \mathrm{Hg}, 95 \% \mathrm{CI}-8.5$ to 2.8 for systolic BP; and $-2.0 \mathrm{~mm} \mathrm{Hg}, 95 \%$ CI -3.5 to 0.4 for diastolic BP). The pooled estimates of intervention effect in all studies were $-5.9 \mathrm{~mm} \mathrm{Hg}$ (95\% CI -7.0 to 4.7 ) for systolic BP and $-2.7 \mathrm{~mm} \mathrm{Hg}(95 \% \mathrm{CI}-3.6$ to 1.7$)$ for diastolic BP. Among seven studies with high risk of bias, one did not measure the fidelity of the intervention. Two used individual randomisation design without blinding study participants, and the randomisation sequences and concealment were unknown. Two studies lacked information on participant blindness, antihypertension medication use, intervention fidelity and had high possibility of selection bias. One study had not provided details regarding the randomisation sequences and concealment, and did not report data on antihypertension medication use and intervention fidelity. One study was reported only in abstract and had unknown information on randomisation and its concealment, intervention fidelity as well as outcome assessment.

The sensitivity analysis with the leave-one-out approach found that the pooled effect estimates changed insignificantly.

\section{Results from meta-regression analysis}

Considering only one study in children and others in adults, the meta-regression was restricted to studies among adults. Univariate regression showed that type of salt reduction strategies was not associated with the BP changes $(p=0.47$ for systolic $\mathrm{BP}$ and $\mathrm{p}=0.06$ for diastolic $\mathrm{BP})$. Further multivariable meta-regression adjusting for other variables including participant's hypertension status and quality of study confirmed the same findings from the univariate analyses $(\mathrm{p}=0.83$ and $\mathrm{p}=0.16$ for systolic and diastolic BP, respectively, table 2 ).

When our analysis was limited to the studies with high quality only, only two types of intervention were left: health education and salt substitute. Both univariate and multivariate analyses found that the type of salt reduction interventions was not associated with BP change.

\section{DISCUSSION \\ Main findings}

This systematic review identified 24 RCTs that assessed the effect of salt reduction strategies in lowering BP in a period of 2 months to 3 years in China. The salt reduction strategies employed in these studies could be classified into four types: health education (8 studies), salt restriction diet (2 studies), salt restriction spoon (1 study) and salt substitute (13 studies). Among them, nine were classified with high quality and eight had data available on changes in BP for the meta-analysis. Our meta-analysis of six studies on salt substitute with high quality showed a significant estimated pooled effect size of $-5.7 \mathrm{~mm} \mathrm{Hg}$ in systolic BP and $-2.0 \mathrm{~mm} \mathrm{Hg}$ in diastolic BP among Chinese adults. One study with high quality on health education showed that a school-based health education programme significantly reduced systolic BP in the parents of school students by $2.3 \mathrm{~mm} \mathrm{Hg}$ on average but a non-significant reduction in systolic and diastolic BP among children and diastolic BP in parents. The meta-regression analysis among all included studies in adults showed that the type of intervention strategy was not significantly associated with the intervention effect on BP after adjusting for baseline hypertension status and quality of study. These results strongly support to promote the use of salt substitute for control of hypertension in China and call for more studies with high quality to provide evidence for other salt reduction strategies.

Table 2 Multivariate meta-regression on the association of intervention effect on blood pressure change with type of intervention strategy, hypertension status and study quality

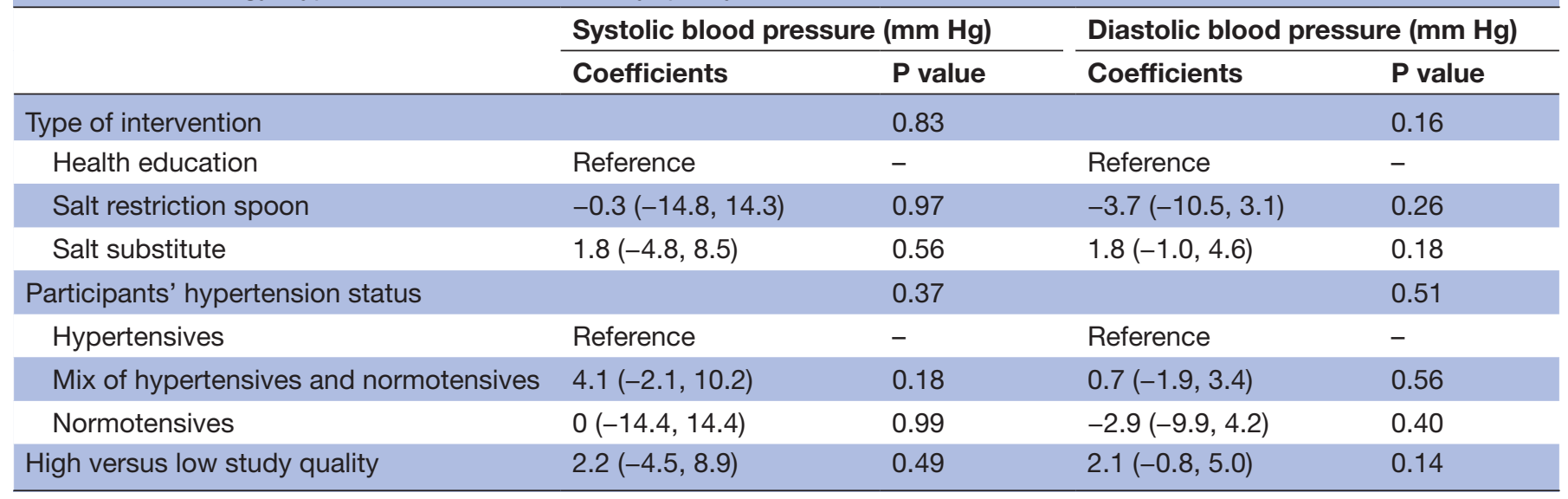




\section{Evidence on effect of different salt reduction strategies}

Among four types of salt reduction strategy evaluated in the review, salt substitute strategy was studied the most intensively, with the highest total number of study (13 studies), total number of study participants (5653 adults) and total number of study with high quality (6 studies with 1694 adults), and the effect size changed very little between all studies and those with high quality. Thus, our meta-analysis on the pooled estimates of intervention effect of salt substitute in lowering BP had a greater power to draw conclusion and the results should be much reliable.

Second to salt substitute, there were seven studies on the effect of health education. However, only three of them judged with high quality, and one had no baseline BP reported. The other two studies were actually of one trial, School-EduSalt, with two different study populations, children and their parents. ${ }^{18}$ The trial used cluster randomised controlled design to test the effect of a school-based health education in lowering the family's salt intake and $\mathrm{BP}$ as the consequence. The intervention successfully reduced salt intake in the family by about $25 \%$ in a school term. ${ }^{18}$ In addition, mean systolic BP was reduced significantly by $2.3 \mathrm{~mm} \mathrm{Hg}$ among family adults. ${ }^{18}$ Compared with the effect size reported in studies with not high quality, this effect size was much smaller. And it was smaller than that reported in studies with high quality evaluating salt substitute. However, compared with the Cochrane review results among non-Chinese populations, the School-EduSalt trial showed much more promising results. ${ }^{29}$ As the evidence was driven from only one trial with high quality, our study urges for future studies to reproduce the effect of this particular strategy before it can be scaled up widely. The CRHI-SRS trial was unique in its design and intervention, and was not included in our meta-analyses because of no data available at the baseline, in which the population mean sodium intake measured by 24 hours' urine was significantly reduced but mean BP was not. ${ }^{12}$ Other health education strategies evaluated in China are lacking high-quality evidence and require future studies with high quality to prove their efficacy.

We also identified two studies on salt restriction diet and one study on salt restriction spoons. All these studies claimed a significant effect of the interventions among either patients with hypertension or on dialysis. However, the quality was all considered low. Moreover, the majority of trials evaluating low-sodium diet were short term and the evidence on long-term intervention effect was lacking.

\section{Comparison of different salt reduction strategies in effect size}

We tried to understand which of the four salt reduction strategies were more effective than the others. The metaanalyses on studies with high quality indicated that the effect by the salt substitutes was greater than that by the school-based health education. However, the studies on salt substitutes were mostly conducted among patients with hypertension but the school-based health education was among children and their parents. Further, the type of intervention strategy was not significantly associated with the net intervention effect in both the univariate and the multivariable meta-regression models with adjustment for the baseline hypertension status and quality of study. The negative results might be due to the small number of studies included and the large heterogeneities among the studies. Anyway, there is not enough power for us to answer this particular question so far.

\section{Limitations}

There are some limitations in our study. First, although four types of salt reduction interventions were tested with randomised controlled design among Chinese and included in our systematic review, we are unable to compare among them to answer which one is more effective than the others. This is mainly due to the poor quality and small number of the studies pertaining to interventions other than salt substitute. Second, the quality of the trials varied widely although all of them used the RCT design. The frequent biases included incomplete reporting of randomisation process, lack of blinding of participants, poor intervention fidelity and lacking information on antihypertension treatment. Besides, the characteristics of trials included in the meta-analysis were varied in the span of age of participants, the spectrum of disease as well as sample sizes of studies. Thus, significant heterogeneity stemmed from the variation in participant demographics, study characteristics, and so on, which has not been explained well. Finally, almost all of the studies included were conducted among hypertensives or the combination of hypertensives and normotensives. The impacts of salt reduction strategies in normotensives in reducing $\mathrm{BP}$ need to be evaluated by further welldesigned studies.

\section{CONCLUSION}

In summary, our study demonstrated that salt reduction by salt substitutes had abundant high-quality evidence on the effect of lowering BP among Chinese adults, particularly in patients with hypertension. The other intervention strategies require further well-designed studies to warrant their efficacy in lowering BP.

Acknowledgements We thank Ms Yi Guo at Yale University School of Public Health for assistance with assessing the quality of studies.

Contributors YW conceived and designed the review. AJ and YW performed the data extraction and preparation. AJ and YW contributed to the assessment of risk of bias. AJ and WX conducted the data analysis. AJ and YW wrote the paper, which was critically reviewed and approved by all the authors. AJ and YW had full access to all the data in the study and take responsibility for the integrity of the data and the accuracy of the data analysis. YW is the guarantor of the study.

Funding The study is funded by the National Key R\&D Program of China (2016YFC1300200).

Competing interests YW has received a small research fund from Chongqing Jiankangyuan Health Technology to study the effect of a new salt substitute with very low sodium.

Patient consent for publication Not required.

Provenance and peer review Not commissioned; externally peer reviewed. 
Data availability statement All data relevant to the study are included in the article or uploaded as supplementary information. All data used in this study are from public literatures.

Open access This is an open access article distributed in accordance with the Creative Commons Attribution Non Commercial (CC BY-NC 4.0) license, which permits others to distribute, remix, adapt, build upon this work non-commercially, and license their derivative works on different terms, provided the original work is properly cited, appropriate credit is given, any changes made indicated, and the use is non-commercial. See: http://creativecommons.org/licenses/by-nc/4.0/.

ORCID iD

Aoming Jin http://orcid.org/0000-0002-7221-5444

\section{REFERENCES}

1 Forouzanfar MH, Liu P, Roth GA, et al. Global burden of hypertension and systolic blood pressure of at least 110 to $115 \mathrm{MM} \mathrm{Hg}, 1990-$ 2015. JAMA 2017;317:165-82.

2 Roth GA, Abate D, Abate KH, et al. Global, regional, and national age-sex-specific mortality for 282 causes of death in 195 countries and territories, 1980-2017: a systematic analysis for the global burden of disease study 2017. The Lancet 2018;392:1736-88.

3 Lawes CMM, Hoorn SV, Rodgers A. Global burden of bloodpressure-related disease, 2001. The Lancet 2008;371:1513-8.

4 Lackland DT, Weber MA. Global burden of cardiovascular disease and stroke: hypertension at the core. Can J Cardiol 2015;31:569-71.

5 Wang Z, Chen Z, Zhang L, et al. Status of hypertension in China: results from the China hypertension survey, 2012-2015. Circulation 2018;137:2344-56.

6 He FJ, Li J, Macgregor GA. Effect of longer-term modest salt reduction on blood pressure. Cochrane Database Syst Rev 2013:Cd004937.

7 Alwan A. Global status report on noncommunicable diseases 2010. Geneva: World Health Organization, 2011.

8 Mozaffarian D, Fahimi S, Singh GM, et al. Global sodium consumption and death from cardiovascular causes. N Engl J Med 2014;371:624-34.

9 Anderson CAM, Appel LJ, Okuda N, et al. Dietary sources of sodium in China, Japan, the United Kingdom, and the United States, women and men aged 40 to 59 years: the INTERMAP study. J Am Diet Assoc 2010;110:736-45.

10 Shuster JJ. Review: cochrane Handbook for systematic reviews for interventions, version 5.1.0, published 3/2011. Julian P.T. Higgins and Sally green, editors. Research Synthesis Methods 2011;2:126-30.

11 Sedgwick P. Meta-Analysis: testing for reporting bias. BMJ 2014;350:g7857.

12 Li N, Yan LL, Niu W, et al. The effects of a community-based sodium reduction program in rural China - a cluster-randomized trial. PLoS One 2016:11:e0166620.

13 Sun GJ, Jiang EL, WH Y, et al. An observational study on the improvement of hypertension in maintenance dialysis patients by strict salt limitation. Chinese Journal of Modern Medicine 2013;23:81-4. (in Chinese).
14 Liberati A, Altman DG, Tetzlaff J, et al. The PRISMA statement for reporting systematic reviews and meta-analyses of studies that evaluate healthcare interventions: explanation and elaboration. BMJ 2009;339:b2700.

$15 \mathrm{Mu}$ J, Liu Z, Liu F, et al. Family-Based randomized trial to detect effects on blood pressure of a salt substitute containing potassium and calcium in hypertensive adolescents. Am J Hypertens 2009;22:943-7.

16 Chen XF, Wang GC, Zeng YG, et al. Application of the transtheoretical model in health education of low salt diet for patients with hypertension. Nursing Research 2011;25:1638-40. (in Chinese).

17 DF W, Li R. Research on the application of motivational interviewing for the promotion of low sodium diet in patients with essential hypertension. Modern Diagnosis and Treatment 2014;25:3372-3. (in Chinese).

18 He FJ, Wu Y, Feng X-X, et al. School based education programme to reduce salt intake in children and their families (School-EduSalt): cluster randomised controlled trial. BMJ 2015;350:h770.

19 Yuan Y, Zhou Y, Wang L, et al. Effect of moving health education on salt restriction intervention in patients with salt sensitive hypertension. Chinese Journal of Social Medicine 2019;36:281-3. (in Chinese).

20 LD H. Application of salt restriction spoon in blood pressure control of patients with hypertension. Contemporary nurse: Academic Edition 2013;7:33-4. (in Chinese).

$21 \mathrm{JH} \mathrm{H}$, YF W, Zhao LC. Effects of salt substitution on blood pressure in hypertensive patients and their families-A randomized doubleblind controlled trial. The Ninth Annual Academic Meeting of Daneng Nutrition Center 2006. (in Chinese).

22 Group CSSSC. Salt substitution: a low-cost strategy for blood pressure control among rural Chinese. A randomized, controlled trial. J Hypertens 2007;25:2011-8.

23 Zhou X, Liu J-X, Shi R, et al. Compound ion salt, a novel low-sodium salt substitute: from animal study to community-based population trial. Am J Hypertens 2009;22:934-42.

24 Zhao X, Yin X, Li X, et al. Using a low-sodium, high-potassium salt substitute to reduce blood pressure among Tibetans with high blood pressure: a patient-blinded randomized controlled trial. PLOS One 2014;9:e110131.

25 ZF L, Yang H, YF W, et al. A randomized controlled study of the relationship between low sodium and potassium-rich salt substitute and blood pressure in the middle-aged and elderly population. Chinese Journal of Geriatrics 2014;33:365-7. (in Chinese).

26 Zhang H, Li D, Li W, et al. Safety of long-term enriched potassium salt consumption and effect on blood pressure in Chinese. Journal of hypertension 2015;33:e148-9.

27 Zhou B, Webster J, Fu L-Y, et al. Intake of low sodium salt substitute for 3years attenuates the increase in blood pressure in a rural population of North China - a randomized controlled trial. Int $J$ Cardiol 2016;215:377-82.

28 Yang GH, Zhou X, WJ J, et al. Effects of a low salt diet on isolated systolic hypertension: a community-based population study. Medicine 2018;97:e0342.

29 Adler AJ, Taylor F, Martin N, et al. Reduced dietary salt for the prevention of cardiovascular disease. Cochrane Database Syst Rev 2014;83:Cd009217. 